\title{
Conseil consultatif de la recherche forestière du Canada Rapport annuel 1991
}

\author{
Mai 1992
}

\section{Résumé}

Ce rapport porte sur l'année civile 1991. Le Conseil consultatif de la recherche forestière du Canada (CCRFC) a été créé en 1983 pour conseiller Forêts Canada sur les priorités et les politiques en matière de recherche forestière. En 1987, le Conseil canadien des ministres de l'Environnement (CCME) a accepté de mettre sur pied des groupes consultatifs de la recherche forestière dans chaque province et territoire et a chargé le CCRFC de coordonner la préparation d'un survol national annuel des priorités et des préoccupations en recherche forestière. Depuis ce temps, le Conseil prépare ce survol national annuel pour le CCMF avec l'aide et l'étroite collaboration des groupes consultatifs de la recherche forestière des provinces et des territoires; ce rapport est publié dans la revue The Forestry Chronicle.

En 1991, Forêts Canada a déposé son premier rapport annuel au Parlement. Le Conseil voit d'un bon oeil ce nouveau processus qui sera très utile pour sensibiliser les députés et le public aux enjeux de la foresterie et à l'importance de la recherche forestière. Pendant l'année, Forêts Canada s'est avancé dans la préparation des énoncés de mission de ses établissements. Ces énoncés sont non seulement importants pour définir le rôle du Ministère, mais le processus lui-même de rédaction est également tout aussi important pour obtenir le soutien des employés et créer un esprit de corps. Le CCRFC est heureux de voir que Forêts Canada prend des initiatives positives dans d'autres domaines, plus précisément: l'inclusion des principaux éléments de recherche dans les nouvelles ententes sur les forêts conclues avec les provinces; le Plan vert et ses éléments portant sur la recherche forestière et les forêts modèles; les progrès accomplis dans le domaine de la biotechnologie; et l'élaboration du programme stratégique de partenariat dans la recherche auquel participent l'industrie et le Conseil de recherches en sciences naturelles et en génie du Canada.

Le Conseil formule les recommandations suivantes:

1. Que Forêts Canada entreprenne ou parraine rapidement une étude portant sur l'offre et la demande de chercheurs dans les diverses disciplines que regroupe la recherche forestière afin de bien définir la rareté apparente de chercheurs en foresterie.

2. Que Forêts Canada offre de plus nombreuses possibilités d'emplois d'été aux étudiants de premier cycle, accorde aux universités son entière collaboration à des programmes conjoints destinés aux élèves de premier et deuxième cycle et améliore ses programmes de «stage» à l'intention des étudiants ayant récemment obtenu un doctorat, par l'attribution, par exemple, de bourses d'études postdoctorales au sein du Ministère et dans les universités. Ces mesures permettront de mieux faire connaître les possibilités qu'offre une carrière de chercheur et d'aider les jeunes chercheurs à s'établir.

3. Que Forêts Canada ait davantage recours au fonds spécial et fort utile qu'il a créé et où il verse un pourcentage relativement faible de son budget afin de donner rapidement suite à de nouvelles pistes de recherche prometteuses.
4. Que Forêts Canada élargisse ses programmes de recherche internes et conjoints destinés à mieux comprendre le fonctionnement de l'écosystème forestier afin de s'assurer que la gestion des ressources forestières du Canada soit conforme aux principes du développement durable.

5. Que Forêts Canada tienne de vastes consultations et envisage de mettre sur pied, au niveau national ou régional, des programmes de recherche conjoints auxquels participeront ses propres employés, les universités, les provinces, l'industrie et les organisations non gouvernementales comme l'Institut de recherches politiques afin d'intensifier la recherche sur la gestion intégrée des ressources forestières, notamment sur ses aspects politiques et socio-économiques.

6. Que Forêts Canada mette davantage l'accent sur l'information technique compte tenu de la demande croissante du public pour de l'information technique sur les forêts et les questions environnementales; le Conseil appuie fortement les efforts actuellement déployés par Forêts Canada pour améliorer son programme de transfert technologique et promouvoir la mise en application des résultats de la recherche.

7. Que Forêts Canada évalue et modifie, dans la mesure du possible, le système de reconnaissance professionnelle des chercheurs pour donner plus d'importance aux activités de transfert technologique, y compris la préparation de publications pratiques du genre «manuel», afin d'encourager les chercheurs à viser la mise en application des résultats qu'ils ont obtenus.

8. Que Forêts Canada s'assure que des groupes consultatifs de recherche existent dans tous ses établissements et élargisse la gamme des intervenants parmi ces groupes consultatifs de recherche et les autres groupes établis aux niveaux régional, provincial et national.

9. Que Forêts Canada continue d'accorder de l'importance au moral de ses employés en les faisant participer à la gestion, qu'il appuie fortement les déplacements et le dialogue entre les établissements ainsi que la participation aux conférences et ateliers scientifiques importants.

10. Que Forêts Canada accroisse les possibilités de congés sabbatiques et de perfectionnement des chercheurs et du personnel technique afin d'empêcher l'apathie de s'installer et de favoriser une plus grande productivité.

11. Que Forêts Canada envisage toutes les possibilités pour mettre en oeuvre un programme similaire au programme américain McIntyre-Stennis afin de favoriser davantage la recherche forestière à long terme dans les universités au Canada.

12. Que Forêts Canada élabore une méthode afin que des critères économiques et sociaux interviennent dans la sélection des projets de recherche et qu'il les incorpore à son processus de planification de la recherche.

Le Conseil tiendra trois réunions en 1992 et se penchera sur les questions suivantes: mise à jour de l'inventaire des projets de recherche forestière entrepris en 1987 par A.J. Kayll; exécution, mécanismes, structures et financement de la recherche par opposition aux processus de planification; 
le document Durabilité des forêts: Un engagement canadien, avec une attention particulière aux aspects de la recherche; autres conclusions à tirer de la rencontre avec les jeunes chercheurs; moyens d'encourager, d'appuyer et de renforcer les groupes consultatifs de la recherche des provinces et des territoires; autres mesures pour renforcer le transfert technologique; programme d'économie forestière de l'administration centrale de Forêts Canada; attitudes et formation de la main-d'oeuvre sylvicole à l'égard des considérations environnementales à retenir dans la planification et l'exécution de leurs tâches quotidiennes; visite du chantier d'une compagnie forestière afin de savoir ce qu'on y pense des besoins de recherche et de connaître leurs vues sur l'établissement des priorités de la recherche et sur la gestion de la recherche et du transfert technologique; et grandes tendances influençant l'avenir de la foresterie et leurs incidences sur la recherche forestière.

\section{Introduction}

Ce rapport annuel du Conseil consultatif de la recherche forestière du Canada (CCRFC) porte sur l'année civile 1991. Le CCRFC, créé en 1983, conseille Forêts Canada, au niveau national, sur les priorités et les politiques de la recherche forestière. En 1987, le Conseil canadien des ministres des forêts (CCMF) a donné son accord à la création de groupes consultatifs de la recherche forestière dans les provinces et les territoires et a chargé le CCRFC de coordonner la préparation d'un survol national des priorités et des préoccupations de la recherche forestière. Le CCRFC et le plaisir d'annoncer que tous les groupes consultatifs fonctionnent maintenant dans toutes les provinces. Avec l'aide et la collaboration étroite des groupes consultatifs provinciaux de la recherche forestière, le Conseil prépare ce survol national annuel pour le CCMF depuis 1987. Ce rapport annuel est publié chaque année dans The Forestry Chronicle, généralement le numéro de février.

En 1991, Forêts Canada a déposé son premier rapport annuel au Parlement. Le Conseil voit d'un bon oeil ce processus qui sera certainement précieux et utile pour sensibiliser les députés et le public aux grands enjeux de la foresterie, y compris à l'importance vitale d'un programme de recherche solide et vigoureux. Il faut applaudir à la décision de faire de la recherche forestière l'un des grands sujets du prochain rapport annuel. Le Conseil était également heureux de constater les progrès sans cesse accomplis dans l'élaboration des énoncés de mission des divers établissements de recherche de Forêts Canada et la participation des employés au processus. Ces énoncés en eux-mêmes sont importants car ils expliquent les rôles du Ministère tant aux organismes externes qu'internes et aident à obtenir le soutien des employés et à créer un esprit de corps, mais le processus en lui-même, lorsqu'il est mené à bien et qu'il accorde toute l'attention nécessaire à une participation du personnel, est tout aussi important. De plus, le CCRFC est heureux de souligner les progrès accomplis par Forêts Canada dans un certain nombre d'autres domaines, plus précisément l'inclusion d'importants éléments de recherche dans la nouvelle génération d'entente de foresterie avec les provinces, le programme des forêts modèles et les éléments de recherche forestière intégrés au Plan vert du gouvernement fédéral; les progrès accomplis dans le domaine de la biotechnologie, et l'élaboration du programme stratégique de partenariat dans dans la recherche auquel participent l'industrie et le Conseil de recherches en sciences naturelles et en génie du Canada.

L'annexe I énumère les membres composant le Conseil en 1991. Trois réunions ont eu lieu au cours de l'année, l'une à Edmonton du 26 au 28 février, la seconde à Petawawa du 7 au 9 mai et la troisième à Toronto les 26 et 27 septembre. M. Jean Claude Mercier a participé à la réunion de Petawawa et les membres du Conseil sont heureux de constater son intérêt indéfectible et sa précieuse contribution. Les membres du Conseil aimeraient également faire savoir aux gestionnaires et au personnel de Forêts Canada qu'ils ont apprécié leur contribution et leur intérêt et souhaiteraient remercier tout particulièrement les employés d'Edmonton et de Petawawa qui n'ont pas ménagé temps et efforts pour expliquer leurs programmes et leurs plans.

\section{Principaux enjeux et préoccupations en 1991}

\section{La relève chez les .chercheurs}

Diverses sources ont fait connaître au Conseil l'existence d'une pénurie apparente de chercheurs canadiens en foresterie, notamment dans certaines disciplines comme la dendrométrie, la sylviculture et les feux de forêt. Par conséquent, le Conseil a prévu de rencontrer un groupe de jeunes chercheurs en foresterie pour savoir ce qui les a poussés à se lancer dans ce domaine et ce qui fait obstacle à leur carrière. Une rencontre d'une demi-journée avec cinq scientifiques a été organisée lors de la première réunion du Conseil en 1991 à Edmonton, en Alberta.

Les cinq chercheurs invités étaient Allison Nicholson, de la Direction de la recherche du ministère des Forêts de la Colombie-Britannique, Ellen MacDonald, de l'École des Sciences forestières de l'Université de l'Alberta, Yves Bergeron, du département de biologie de l'Unviersité du Québec à Montréal, Gina Mohammed, de l'Institut de recherche forestière de l'Ontario à Sault Ste. Marie, et David Langor de la Région du Nord-Ouest de Forêts Canada. Fait intéressant à noter, aucun d'eux n'avait obtenu leur diplôme universitaire de premier cycle en foresterie.

On avait demandé aux chercheurs invités à participer à cette réunion de réfléchir à une série de questions et d'être prêts à en discuter. Les questions étaient les suivantes:

- Pourquoi le Canada connaît-il une pénurie de certains types de chercheurs en foresterie?

- Pourquoi faites-vous de la recherche forestière?

- Quelles frustrations avez-vous éprouvées ou éprouvezvous à titre d'étudiant diplômé?

- Quelles frustrations éprouvez-vous ou pensez-vous devoir affronter à titre de chercheur actif?

- Quels mécanismes poussent les étudiants vers la recherche forestière et ces mécanismes sont-ils suffisants?

- Dans quelle mesure vos emplois d'été ont-ils influencé votre choix de carrière?

- Y-a-t-il quelqu'un qui a influencé de façon marquée votre carrière?

La discussion sur les facteurs motivant les étudiants à envisager une carrière de chercheur a été animée et a mis en lumière plusieurs faits intéressants. La pénurie de chercheurs qualifiés en foresterie est bien réelle dans certaines disciplines, mais il est certain que de nombreux domaines se rattachant à la foresterie connaissent un surplus de spécialistes. La situation varie d'une discipline à l'autre et 
peut changer très rapidement dans le temps. Il y a peu de perspectives d'emploi dans certaines disciplines, ce qui détourne les étudiants d'une carrière en recherche forestière. Pour un scientifique récemment diplômé, la recherche d'un emploi est une opération d'infiltration d'un réseau en grande partie tributaire de contacts informels, du bouche à oreille et des contacts de son professeur.

Plusieurs raisons expliquent pourquoi les gens n'entreprennent pas de carrière scientifique. Bon nombre d'entre eux ne comprennent pas la valeur de la science et considèrent que ce type de carrière se déroule dans l'ombre. Les professeurs de premier cycle peuvent faire toute la différence. Les cours de premier cycle ne soulignent souvent pas l'importance de la recherche, les lacunes des connaissances et l'excitation et le défi qu'offre la recherche.

Les facultés de foresterie ne semblent pas bien promouvoir les carrières de chercheurs en foresterie. D'autres facultés et écoles donnent en apparence l'impression d'aborder des problèmes écologiques et environnementaux importants et attirent ainsi de bons étudiants. Le manque de soutien financier offert aux étudiants diplômés des facultés de foresterie est un autre facteur important. Un emploi d'été fructueux dans un domaine de la recherche a souvent une influence déterminante dans le choix d'une carrière scientifique.

Les scientifiques ont identifié plusieurs obstacles entravant une carrière en recherche et la mise en application des résultats. Trop souvent, un financement à court terme ne pousse pas les chercheurs à s'attaquer aux problèmes de foresterie difficiles qui exigent des travaux de longue haleine et leur permettent de perfectionner leurs compétences scientifiques. Le système de reconnaissance professionnelle est quelquefois capricieux et lent à réagir de sorte que de bons projets de recherche offrant d'excellentes perspectives de résultats passionnants et utiles ne sont pas nécessairement appuyés par une augmentation des ressources; les précieux efforts qu'il faut déployer pour amener un projet au stade pratique peuvent ne pas être reconnus et récompensés. Forêts Canada a eu une heureuse initiative en mettant sur pied, à l'administration centrale, un fonds spécial accessible aux établissements de recherche sur demande et destiné à fournir rapidement du financement à de nouveaux projets de recherche innovateurs.

Recommandation 1. Que Forêts Canada entreprenne ou parraine rapidement une étude portant sur l'offre et la demande de chercheurs dans les diverses disciplines que regroupe la recherche forestière afin de bien définir la rareté apparente de chercheurs en foresterie.

Recommandation 2. Que Forêts Canada offre de plus nombreuses possibilités d'emplois d'été aux étudiants de premier cycle, accorde aux universités son entière collaboration à des programmes conjoints destinés aux élèves de premier et deuxième cycles, et améliore ses programmes de «stage» à l'intention des étudiants ayant récemment obtenu un doctorat, par l'attribution, par exemple, de bourses d'études postdoctorales au sein du Ministère ainsi que dans les universités. Ces mesures permettront de mieux faire connaître les possibilités qu'offre une carrière de chercheur et d'aider les jeunes chercheurs à s'établir.

Recommandation 3. Que Forêts Canada ait davantage recours au fond spécial et fort utile qu'il a créé et où il verse un pourcentage relativement faible de son budget afin de donner rapidement suite à de nouvelles pistes de recherche prometteuses.

\section{Fonctionnement de l'écosystème et gestion intégrée des} ressources forestières

Le Conseil reconnaît que l'élaboration d'une gestion véritablement intégrée et équilibrée de toutes les ressources du milieu forestier a progressé, mais il doit souligner qu'il reste encore beaucoup à faire. Sur le plan biologique, la complexité écologique des forêts est lentement élucidée par la recherche effectuée par les universités, les provinces et Forêts Canada; il reste néanmoins beaucoup à apprendre.

Sur le plan socio-économique, la situation est plus compliquée parce que le gestionnaire de la ressource forestière est aux prises avec des attentes souvent divergentes et en constante évolution à l'égard des forêts du pays. Les progrès dans ce domaine semblent moins évidents. Ces dernières questions exigent une recherche de pointe sur la politique publique et le Conseil a peur que trop peu soit fait et que des décisions soient prises sans que les données pertinentes aient été recueillies et analysées minutieusement. Il est vrai que ce genre de décisions doit tenir compte des opinions politiques et d'autres considérations, mais une recherche approfondie, une analyse minutieuse des données et l'élaboration et l'évaluation de solutions de rechange accroissent les chances de faire des choix judicieux.

Recommandation 4. Que Forêts Canada élargisse ses programmes de recherche internes et conjoints destinés à mieux comprendre le fonctionnement de l'écosystème forestier afin de s'assurer que la gestion des ressources forestières du Canada soit conforme aux principes du développement durable.

Recommandation 5. Que Forêts Canada tienne de vastes consultations et envisage de mettre sur pied, au niveau national ou régional, des programmes de recherche conjoints auxquels participeront ses propres employés, les universités, les provinces, l'industrie et les organisations non gouvernementales comme l'Institut de recherches politiques afin d'intensifier la recherche sur la gestion intégrée des ressources forestières, notamment sur ses aspects politiques et socio-économiques.

\section{Transfert technologique}

Le transfert des résultats de recherche aux praticiens de la foresterie sur le terrain est un problème incessant. Les jeunes chercheurs ont eux aussi relevé ce sujet qui semble revenir dans presque toutes les discussions portant sur la recherche forestière. Comme nous l'avons noté précédemment, le système de reconnaissance professionnelle des chercheurs ne récompense pas toujours les efforts déployés pour mettre en pratique les résultats de la recherche. La mise en application de résultats de la recherche est souvent à double sens, ce qui est très souvent ignoré. D'une part, les chercheurs ont souvent de la difficulté à transférer les résultats de la recherche à l'utilisateur et, d'autre part, les utilisateurs sont souvent peu enclins à définir leurs problèmes et besoins et à chercher des scientifiques et des résultats de recherche qui peuvent leur être utiles. On pourra améliorer les processus en motivant à la fois les scientifiques et les praticiens. 
Le Conseil fait l'éloge des efforts incessants de Forêts Canada dans ce domaine. L'atelier interne sur le transfert technologique qui s'est déroulé à Ottawa en novembre 1991 aidera à sensibiliser les chercheurs et à encourager de nouvelles initiatives au sein de Forêts Canada. Il faut élargir le débat et amener une plus vaste gamme d'intervenants à faire connaître leurs préoccupations et leurs idées. Le Conseil aimerait à nouveau faire ressortir l'importance de sa recommandation de l'an dernier, à savoir "Que Forêts Canada fasse en sorte qu'une large gamme d'intéressés participent à son processus consultatif sur la recherche forestière et qu'il encourage les autres organismes à faire de même». Cette recommandation visait tous les aspects du mandat de Forêts Canada, mais il est tout particulièrement important de s'assurer que les utilisateurs des résultats de recherche participent à la définition des objectifs de la recherche.

Un autre aspect important du transfert technologique est le fait que la population est de plus en plus sensibilisée aux enjeux de l'environnement et des ressources naturelles et a besoin d'une bonne information technique. Les efforts de transfert technologique ne devraient pas négliger le volet éducation du public.

Recommandation 6. Que Forêts Canada mette davantage l'accent sur l'information technique compte tenu de la demande croissante du public pour de l'informations techniques sur les forêts et les questions environnementales; le Conseil appuie fortement les efforts actuellements déployés par Forêts Canada pour améliorer son programme de transfert technologique et promouvoir la mise en application de résultats de la recherche.

Recommandation 7. Que Forêts Canada évalue et modifie, dans la mesure du possible, le système de reconnaissance professionnelle des chercheurs pour donner plus d'importance aux activités de transfert technologique, y compris la préparation de publications pratiques du genre «manuel», afin d'encourager les chercheurs à viser la mise en application des résultats qu'ils ont obtenus.

Recommandation 8. Que Forêts Canada s'assure que des groupes consultatifs de recherche existent dans tous ses établissements et élargisse la gamme des intervenants parmi ces groupes consultatifs de recherche et les autres groupes établis aux niveaux régional, provincial et national.

\section{Relations avec le personnel}

Dans toute organisation, le moral du personnel joue un rôle très important dans le fonctionnement adéquat et efficace et l'atteinte des objectifs fixés. C'est particulièrement vrai au sein d'un organisme de recherche où la majeure partie du travail est, du moins dans une certaine mesure, autodirigée. Lors des visites qu'il a effectuées au cours des dernières années dans des établissements de recherche de Forêts Canada, le Conseil a relevé des variations dans le moral des employés. Le moral était toujours très bon lorsque le personnel professionnel participait de près à l'identification des objectifs et à la prise de décisions opérationnelles. Le soin que prenaient ces établissements à "cultiver» leurs relations avec leurs employés avait des effets positifs et les gens aimaient y travailler.

Les gestionnaires peuvent améliorer énormément le moral du personnel de recherche en faisant tout en son pouvoir pour réduire le facteur d'isolement. Dans une organisation ouverte comme Forêts Canada, par opposition à un groupe de recherche confiné à un secteur, les discussions et les contacts avec des collègues et des pairs et avec des gens de domaines scientifiques connexes font avancer la recherche. Il est très important que les chercheurs de Forêts Canada de différents endroits aient des contacts afin de réduire concrètement le déboublement des efforts et la prolifération d'approches non coordonnées à des problèmes communs. Il est également important d'avoir des relations avec les universités et les laboratoires de l'industrie qui seront d'autant facilitées qu'un établissement de Forêts Canada est situé près d'un autre groupe de recherche ou d'un campus. Il est essentiel de prévoir des frais de déplacement pour se rendre à des conférences et effectuer des visites dans d'autres établissements de recherche et de favoriser les appels interurbains et les conférences téléphoniques notamment, mais non pas uniquement, dans les endroits éloignés. Dans un même ordre d'idée, il ne faut pas négliger la valeur des congés sabbatiques et des cours d'une brève durée.

Recommandation 9. Que Forêts Canada continue d'accorder de l'importance au moral de ses employés en les faisant participer à la gestion, qu'il appuie fortement les déplacements et le dialogue entre les établissements ainsi que la participation aux conférences et ateliers scientifiques importants.

Recommandation 10. Que Forêts Canada accroisse les possibilités de congés sabbatiques et de perfectionnement des chercheurs et du personnel technique afin d'empêcher l'apathie de s'installer et de favoriser une plus grande productivité.

\section{Recherche forestière dans les universités}

Depuis quelques années, Forêts Canada versait un «financement global» à la recherche dans les facultés et écoles de foresterie du Canada. L'une des principales caractéristiques de ce programme était sa flexibilité et il était particulièrement utile parce que les doyens pouvaient utiliser les fonds de diverses façons pour appuyer la recherche forestière. D'autre part, le programme était limité puisqu'il ne versait des fonds qu'aux écoles de foresterie.

Aux États-Unis, la recherche forestière effectuée dans les universités est financée depuis 1962 par le McIntyre-Stennis Co-operative Forestry Research Program. Ce programme, crée par la Loi McIntyre-Stennis du Congrès américain, crée un partenariat entre le gouvernement fédéral et les États en vue d'appuyer la recherche forestière dans des universités désignées. La part de financement du fédéral est déterminée par une formule qui permet de verser uniquement des fonds là où l'économie des ressources naturelles et l'engagement envers la recherche justifient l'investissement; les États versent une contribution égale. Ce programme a constitué une source stable et importante de financement de la recherche universitaire; il est de plus très souple d'application et facile à gérer.

Un programme similaire pourrait être mis en oeuvre au Canada soit par l'adoption d'une loi ou l'approbation du Cabinet. Il permettrait d'améliorer la qualité et la quantité de la recherche forestière effectuée dans les universités, aiderait à former les scientifiques nécessaires pour réaliser des recherches de haut calibre et permettrait aux étudiants ayant obtenu un baccalauréat d'être davantage exposé à un climat 
de recherche intense et les rendrait plus enclins à utiliser dans leur travail quotidien les résultats de recherche.

Recommandation 11. Que Forêts Canada envisage toutes les possibilités pour mettre en oeuvre un programme similaire au programme américain McIntyre-Stennis afin de favoriser davantage la recherche forestière à long terme dans les universités au Canada.

\section{Changement climatique}

Le Conseil a été heureux d'apprendre que le programme de recherche de Forêts Canada mettrait davantage l'accent sur le changement climatique. Le changement climatique a des incidences énormes sur la gestion des ressources forestières du Canada et il est important que ces incidences soient évaluées et que des solutions d'aménagement durable soient élaborées le plus rapidement possible.

\section{Recherche sur l'économie et la politique forestières}

Le Conseil attache beaucoup d'importance à la recherche sur l'économie et les politiques et, comme il a été mentionné précédemment, il est persuadé qu'il faudrait intensifier la recherche visant à mettre au point des solutions et des méthodes de gestion intégrée des ressources forestières afin qu'elles procurent au pays toute la gamme des avantages possibles. Un autre aspect important est l'élaboration et l'utilisation de critères économiques et sociaux pour la sélection des projets de recherche forestière et la gestion des programmes de recherche. Forêts Canada a bien sûr accompli des progrès appréciables dans l'élaboration de son processus de planification de la recherche et a tenu le Conseil au courant de cette progression, mais le Conseil estime qu'il devrait en faire davantage afin que des considérations d'ordre économique et social interviennent dans le processus de sélection des programmes et des projets de recherche.

Recommandation 12. Que Forêts Canada élabore une méthode afin que des critères économiques et sociaux interviennent dans la sélection des projets de recherche et qu'il les incorpore à son processus de planification de la recherche.

\section{Priorités de la recherche forestière au Canada: Survol}

Le troisième survol annuel des priorités de la recherche forestière au Canada a été compilé pour le CCMF, comme le précise l'introduction. Le président du CCRFC, Jack Toovey, a présenté les résultats de ce survol lors de la réunion du CCMF qui s'est tenue à Ottawa en octobre 1991. Le rapport a été publié dans le numéro de février 1992 de The Forestry Chronicle.

Aux fins de ce survol, les groupes consultatifs de la recherche forestière des provinces et des territoires devaient identifier leurs cinq grandes priorités de recherche forestière. Les priorités qu'ils ont identifiées ont été regroupées en une liste de 10 sujets de recherche qui sont présentés ci-dessous par ordre décroissant d'importance:

- Systèmes de gestion intégrée des ressources et d'appui à la décision.

- Lutte contre les ravageurs et la végétation concurrente et solutions de rechange aux produits chimiques.

- Effets de l'aménagement forestier sur l'environnement.
- Connaissances écologiques en vue d'un aménagement intensif des forêts.

- Données sur la croissance et le rendement.

- Augmentation de la productivité, de l'amélioration des arbres et de la rénégération.

- Inventaires forestiers et classification des stations.

- Méthodes de sylviculture et de récolte et réduction des coûts.

- Gestion des incendies de forêts et méthodes de lutte.

- Aménagement des peuplement mixtes.

La recherche sur la transformation du bois et la mise au point de nouveaux produits a également été reconnue comme importante, mais ce domaine n'est pas la priorité du CCRFC et il n'a donc pas été coté. Les systèmes modernes de traitement des données, l'intelligence artificielle et la biotechnologie ont aussi été jugés importants, mais il s'agit d'outils de recherche plutôt que de sujets de recherche forestière, et ils sont également absents du classement.

Contrairement aux années précédentes, cette liste de priorités a révélé la crainte que cet ordre de priorité n'évolue plus rapidement que ne peuvent le faire les programmes de recherche. Toutefois, aucun des sujets prioritaires de 1991 n'est nouveau. La façon dont ils sont énoncés et leur groupement peuvent être différents en 1991, mais ils figuraient tous dans le survol des priorités de la recherche forestière au Canada de 1990 et ont été traités comme tels par la plupart des provinces et territoires. En demandant à chaque province et territoire de n'identifier que leurs cinq grandes priorités en 1991, nous avons fait converger l'attention vers les grands problèmes et ainsi réduit la longueur de la liste.

Tous sont d'avis que très peu de sujets de recherche reçoivent un financement suffisant dans les régions et que l'attention accordée aux effets sur l'environnement de l'aménagement forestier souffre d'un sous-financement chronique. Les ententes fédérales-provinciales sur les forêts sont essentielles au soutien de la recherche. Ce soutien sera renforcé si l'on met davantage l'accent sur les programmes coopératifs et l'approche concertée devant les organismes de financement. Le regroupement de sources spéciales de financement provenant de l'industrie et des gouvernements et leur gestion conjointe offrent une autre possibilité, au même titre que la réattribution des fonds consacrés à des travaux d'importance moindre, si possible.

Douze problèmes qui peuvent influer sur les priorités de la recherche ont été identifiés:

- Préoccupation et activités canadiennes et internationales à l'égard de la foresterie durable et de l'environnement.

- Aménagement tenant compte des valeurs non ligneuses et diminution des superficies consacrées à la production de bois.

- Mondialisation des marchés, augmentation de la concurrence et opinion publique internationale.

- Sous-financement de la foresterie et fin des ententes fédérales-provinciales.

- Besoin de modèles plus précis en aménagement forestier durable.

- Besoins de meilleures données sur l'importance socioéconomique des forêts.

- Réclamations territoriales et sylviculture sur les terres indiennes.

- Aménagement plus intensif de la forêt boréale mixte.

- Économie des industries secondaires rentables du secteur forestier. 
- Besoin d'information croissant à tous les niveaux.

- Nouvelles politiques provinciales sur la foresterie et la protection des forêts.

- Sylviculture sur les terres agricoles pour le bois et les rideaux-abris.

Certains de ces sujets figuraient sur les listes précédentes des priorités de la recherche, comme la sylviculture à petite échelle, et la foresterie sur les terres indiennes. Ils ne se retrouvent toutefois par sur la liste des priorités de cette année parce qu'ils ne faisaient pas partie des cinq grandes priorités des provinces et des territoires. Ces sujets et d'autres préoccupations, dont bon nombre sont de nature locale. doivent toutefois figurer dans l'assemblage final des projets de recherche entrepris par tout organisme.

Les ministres semblent apprécier à sa juste valeur le survol annuel des priorités de la recherche qui leur est présenté lors de leur réunion annuelle de l'automne. Le CCRFC continuera de collaborer avec les provinces et les territoires afin de préparer et d'améliorer les prochaines versions.

\section{Mesures découlant du Rapport du Conseil de 1990}

Cette année encore, Forêts Canada a remis à la fin de l'année un rapport au Conseil énumérant les mesures qu'il a prises en réponse aux recommandations du CCRFC de l'année précédente. Les membres du Conseil sont fort heureux de la collaboration persistante de Forêts Canada. Ce rapport figure à l'Annexe II.

\section{Plan d'action pour 1992}

Le Conseil tiendra trois réunions régulières en 1992, soit une à Ottawa en janvier, une deuxième à Montréal en mai et une troisième à Vancouver en septembre. Pendant l'année, le Conseil mettra l'accent sur:

1. La mise à jour de l'inventaire de la recherche forestière entrepris en 1987 par A.J. Kayll.
2. L'élaboration, par Forêts Canada, d'un «Programme de science et de technologie", qui portera entre autres sur l'exécution, les mécanismes, les structures et le financement de la recherche par opposition aux processus de planification.

3. L'examen du document Durabilité des forêts: un engagement canadien en tenant tout particulièrement compte du volet de la recherche.

4. Le suivi à donner aux conclusions découlant de la réunion avec les jeunes chercheurs.

5. L'étude des moyens possibles d'encourager, de soutenir et de renforcer les groupes consultatifs de recherche des provinces et des territoires grâce à une participation accrue aux activités du CCRFC, quand les circonstances s'y prêtent.

6. L'étude de mesures supplémentaires à prendre pour renforcer le transfert technologique.

7. L'examen du programme d'économie forestière de l'administration centrale de Forêts Canada.

8. L'étude des attitudes et de la formation des travailleurs forestiers et des façons d'arriver à les modifier pour que la planification et l'exécution des tâches quotidiennes tiennent compte de considérations environnementales.

9. La visite d'un chantier d'une compagnie forestière afin de savoir ce qu'on y pense des besoins de recherche et de connaître leurs opinions sur l'établissement des priorités de la recherche et sur la façon de formuler et de gérer les projets de recherche et le transfert technologique.

11. L'examen des grandes tendances influençant la foresterie et évaluation de leurs incidences sur les priorités de la recherche forestière.

12. La création et le resserrement des liens avec d'autres groupes comme le Conseil de recherches en sciences naturelles et en génie (CRSNG), le Conseil national de recherches, le ministère de l'Industrie, des Sciences et de la Technologie et bien d'autres.

\section{Annexe I}

Membres du Conseil en 1991

\section{Représentants des provinces}

M. T.E. Baker

Direction de la recherche

Ministère des Forêts

31 Bastion Square

Victoria (Colombie-Britannique)

V8W 3E7

M. C. David Rannard

Directeur de la foresterie

Ministère des Ressources naturelles

300-530 Kenaston Boulevard

Winnipeg (Manitoba)

R3N 1 Z4

\section{John Goodman}

Sous-ministre adjoint

Services corporatifs

Ministère des Ressources naturelles de l'Ontario

Whitney Block, $6^{\mathrm{e}}$ étage

Queen's Park

99 Wellesley Street West

Toronto, Ontario

M7A 1W3
M. Jean-Guy Davidson

Tél. : (604) 387-6720

Téléc. : (604) 387-0046

Tél. : (204) 945-7998

Téléc. : (204) 489-1360

Tél. : (416) 965-1897

Téléc. : (416) 314-1910

du développement

Ministère des Forêts

Sainte-Foy (Québec)

G1P 3W8

M. Bernard W. Burgess

Hillcrest Road

R.R. 3

Brockville (Ontario)

K6V 5T3

(président)
Directeur de la recherche et

2700, rue Einstein, Block B.1.185

\section{Représentants de l'industrie}

M. Jack W. Toovey, R.P.F.

Tél. : (604) 985-8680

Téléc. : (604) 985-8528
Tél. : (418) 643-7994

Tél. : (613) 345-2285

Téléc. : (416) 488-5262
Téléc. : (418) 643-2165

2976 William Avenue

North Vancouver (Colombie-Britannique)

V7K 1 Z6

M. Michael R. Innes

Aménagiste forestier

Abitibi-Price Inc. 
207 Queen's Quay West

C.P. 102

Toronto (Ontario)

M5J 2P5

M. Robert W. Udell

Surintendant de la

planification stratégique

Weldwood of Canada Limited

Hinton Division

760 Switzer Drive

Hinton (Alberta)

T7V 1 V7

\section{Représentant de l'Institut forestier du Canada}

M. William J. Brown

Tél. : (705) 789-9647

Directeur général

Algonquin Forestry Authority

22 Main Street

Huntsville (Ontario)

P0A $1 \mathrm{~K} 0$

\section{Représentants des universités}

M. Peter J. Murphy

Faculté d'agriculture et de foresterie

Département de Sciences forestières

Université de l'Alberta

Edmonton (Alberta)

T6G $2 \mathrm{H} 1$

M. Gilles Frisque

Directeur (vice-président)

Tél. : (418) 657-3551

Tél. : (403) 492-2359

Téléc. : (403) 492-4323

Rés : (403) 458-9706

Centre de recherche forestière multirégionale

Université du Québec

2875, boul. Laurier, $3^{\mathrm{e}}$ étage

Sainte-Foy (Québec)

G1V 2M3

Représentant de l'Association canadienne des producteurs de pâtes et papiers

M. Gérald Lapointe

Division des bois et forêts

Tél. : (514) 866-6621

Téléc. : (514) 866-3035
Association canadienne des

producteurs de pâtes et papiers

1155 , rue Metcalfe, $19^{\mathrm{c}}$ étage

Montréal (Québec)

H3B 4T6

Fédération canadienne de la nature

M. Paul Griss

Tél. : (613) 238-6154

Directeur exécutif

Téléc. : (613) 230-2054

Fédération canadienne de

la nature

453 Sussex Drive

Ottawa (Ontario)

KIN 6 Z8

\section{Membre ordinaire}

M. Claude Godbout, Doyen

Tél. : (418) 656-2116

Faculté de foresterie et

Téléc. : (418) 656-3177

de géomatique

Université Laval

Québec (Québec)

G1K 7P4

\section{Membre d'office}

M. Fred C. Pollett

Directeur des Sciences

et du Développement durable

Forêts Canada

Place Vincent-Massey

351 , boul. Saint-Joseph

Hull (Québec)

K1A $1 G 5$

Tél. : (819) 997-1107

Téléc. : (613) 990-3437

\section{Secrétaire administratif}

M. R.J. Bourchier

Tél. : (819) 827-0903

R.R. 1 ,

C.P 255

Cantley (Québec)

JOX $1 \mathrm{LO}$

\section{Annexe II \\ Commentaires de Forêts Canada à l'égard des recommandations de 1990 du Conseil consultatif de la recherche forestière du Canada}

Recommandation 1. Que Forêts Canada fasse en sorte qu'une large gamme d'intéressés participe à son processus consultatif sur la recherche forestière et qu'il encourage les autres organismes à faire de même.

Réponse. Forêts Canada est en principe d'accord avec cette recommandation.

Mesure prise. La recherche sur l'aménagement forestier et la pratique de cette activité ont maintenant recours à une approche plus globale en réponse aux préoccupations du public et à la faveur d'une sensibilisation grandissante, au sein du secteur de forêts, au fait que toutes les utilisations et les valeurs des forêts et des terrains forestiers doivent être prises en compte afin d'arriver à obtenir un développement durable. Le programme de recherche de Forêts Canada est de plus en plus axé sur des questions comme le fonctionnement de l'écosystème et la gestion intégrée des ressources et cette tendance sera encore plus marquée lorsque les projets du nouveau Plan vert seront entièrement opérationnels. Il est clair que les processus de consultation sur la recherche forestière doivent s'adapter à ces mêmes tendances en élargissant la gamme des intervenants; de tels changements commencent à se produire, mais Forêts Canada est conscient qu'il a encore beaucoup à faire à cet égard. Pour atteindre un tel objectif, il faudra toutefois obtenir le soutien et la participation active de tous les grands organismes de foresterie. Cette question doit, croyons-nous, être abordée dans le cadre d'un processus de consultation multi-organismes sur la recherche auquel a souscrit le CCMF en 1987; Forêts Canada ne pourra la résoudre seule. 
Recommandation 2. Que Forêts Canada recherche toutes les occasions pour corréler ses travaux de recherche et les systèmes de classement des stations et qu'il élargisse ainsi l'emploi des résultats de la recherche de façon à maximiser leurs applications et à en prévenir l'application à mauvais escient à des situations mal choisies.

\section{Réponse. Forêts Canada accepte cette recommandation.}

Mesure prise. Depuis quelques années, des progrès importants ont été accomplis en matière d'élaboration, de mise en application et d'intégration de systèmes de classement des stations forestières d'un bout à l'autre du pays. Un certain nombre de manuels pratiques ont été préparés pour aider les aménagistes à faire le lien entre ces systèmes de classement et les pratiques opérationnelles. Les travaux sur ces systèmes se poursuivent pour les rendre aussi accessibles et compatibles que possible, en grande partie sous la direction du groupe de travail sur la recherche relative à la classification des stations de Forêts Canada. Les activités sur la classification des stations seront étoffées par un certain nombre d'activités du Plan vert, y compris l'intensification des efforts de recherche et, plus particulièrement, grâce à l'application directe des principes et systèmes de classement des stations dans le réseau prévu de forêts modèles.

Recommandation 3. Que Forêts Canada étoffe ses rapports avec les universités et les incite à faire davantage pour élargir leur quête de diplômés en insistant sur les défis très réels lancés par la foresterie. Que Forêts Canada envisage également de détacher de ses scientifiques dans les universités pour deux ou trois ans, afin de corriger la pénurie de compétences spécifiques et d'augmenter la formation de spécialistes dans leurs disciplines.

\section{Réponse. Forêts Canada accepte cette recommandation.}

Mesure prise. Ces dernières années, Forêts Canada a pris un certain nombre de mesures destinées à renforcer la recherche forestière dans les universités canadiennes, tant dans les facultés de foresterie que dans les autres départements connexes. Dés initiatives qui ont été prises ou appuyées par Forêts Canada, comme le programme de réseaux de centres d'excellence, d'autres réseaux de recherches spécialisées comme Biocide et Microbionet, l'énoncé de la mission du CRSNG en matière de foresterie et le programme de partenariat de recherche Forêts Canada/CRSNG (voir cidessous), ont toutes été conçues pour intensifier la recherche universitaire dans des sujets forestiers fortement prioritaires. Forêts Canada a également continué de collaborer avec les universités, et de leur donner son appui, grâce à des mécanismes comme des postes de professeurs auxiliaires, un programme de supervision des étudiants diplômés de premier cycle et des conférences occasionnelles destinées à enrichir le contenu des cours. Forêts Canada a toujours consenti à affecter à plus long terme des chercheurs dans les universités pour des besoins particuliers, et est toujours disposé à le faire, mais juge qu'il est important que de telles ententes ne viennent pas se substituer pour de bon au développement du savoir par le système universitaire lui-même. Nous convenons, à l'instar du CCRFC, que les universités devraient déployer davantage d'efforts pour attirer en foresterie des étudiants détenant un diplôme de premier cycle (et que Forêts Canada devrait les aider le cas échéant), mais nous sommes persuadés que cela ne se produira que dans la mesure où elles seront capables de se doter et de conserver un savoir reconnu à l'échelle mondiale et d'obtenir une reconnaissance dans des domaines particuliers de la foresterie ou de sujets connexes.

Recommandation 4. Que Forêts Canada s'efforce pleinement de trouver d'autres moyens et d'autres techniques pour accentuer les programmes de collaboration, selon le modèle de partenariat dans la recherche appliquée avec le CRSNG.

Réponse. Forêts Canada accepte cette recommandation.

Mesure prise. Forêts Canada est heureux du succès initial de son programme de partenariat avec le CRSNG et de la réaction de l'industrie à cet égard. En 1992, Forêts Canada examinera attentivement la possibilité de conclure des ententes similaires avec d'autres organismes comme le Conseil de recherches en sciences humaines (CRSH). Simultanément, Forêts Canada examinera de près les points forts et les points faibles de l'ensemble de ses mécanismes de recherche conjointe, dans le cadre d'une initiative stratégique plus vaste concernant les rôles et responsabilités en matière de recherche.

Recommandation 5. Que, en instituant des programmes de recherche commune avec l'industrie, Forêts Canada fasse en sorte que les sociétés participantes soient pleinement conscientes des mesures nécessaires pour obtenir un traitement fiscal favorable pour leur participation.

Réponse. Forêts Canada est persuadé que les chefs de ces entreprises devraient être informés des modalités leur permettant d'obtenir un traitement fiscal favorable, mais qu'il revient à chaque compagnie d'en prendre l'initiative et de s'informer auprès de Revenu Canada.

\section{Mesure prise. Non disponible.}

Recommandation 6. Que Forêts Canada examine bien son programme de recherche en économie et en politique forestières afin d'évaluer son à-propos et le caractère suffisant des liens noués avec les provinces, l'industrie et les universités. Qu'une révision approfondie du programme soit effectuée sur le sujet, la documentation complète sur le programme devant être réunie et mise à la disposition du Conseil ainsi que des clients et des collaborateurs de Forêts Canada.

Réponse. Forêts Canada accepte cette recommandation.

Mesure prise. La direction de la vérification et de l'évaluation du Ministère effectue une évaluation en profondeur du programme de recherche en économie forestière de Forêts Canada. Les résultats de cette évaluation et les réactions de Forêts Canada à toute recommandation qui pourra être formulée seront mis à la disposition du CCRFC et des autres parties intéressées. Simultanément, Forêts Canada a pris un certain nombre de mesures pour renforcer les liens entre son programme de recherche et son programme sur l'économie. 
Parmi les mesures prises, mentionnons la participation de la Direction des politiques et de l'économie aux composantes appropriées du Plan vert et à d'autres activités relatives à l'environnement et au développement durable. Il a également créé un groupe de travail sur la recherche en économie forestière.

Recommandation 7. Qu'on s'efforce sérieusement d'augmenter la notoriété des deux instituts spécialisés de Forêts Canada de façon à faire davantage connaître leur rôle, notamment quant aux préoccupations écologiques auprès des cercles de forestiers et d'écologistes. Un changement de nom pourrait être envisagé. À la lumière des suggestions des scientifiques et du personnel, qu'on recherche des dénominations qui insisteraient davantage sur la notion de développement durable et d'équilibre écologique.

Réponse. Forêts Canada accepte cette recommandation en principe.

Mesure prise. Tous les établissements de Forêts Canada, y compris, mais non pas uniquement, ses deux instituts, se préoccupent constamment du profil et de la qualité du programme de recherche du Ministère. Comme l'indiquait la réponse à la recommandation $\mathrm{n}^{\circ} 1$, le programme de recherche de Forêts Canada met maintenant beaucoup plus l'accent sur les questions relatives à l'environnement et au développement durable, et il est capital que ses clients et ses collaborateurs le comprennent bien. Les scientifiques participent activement à l'élaboration, à la coordonnation et la gestion des nouvelles initiatives dans ces domaines. L'initiative stratégique sur les rôles et responsabilités en matière de recherche dont nous avons parlé précédemment aborde également les questions de la qualité et du profil scientifique tant au sein de Forêts Canada que dans l'ensemble du secteur forestier en général. Dès qu'ils seront disponibles, les résultats de cette étude, qui porte en partie sur les rôles respectifs et les noms des établissements régionaux et des instituts de Forêts Canada, seront transmis au CCRFC afin qu'il puisse en discuter et formuler ses commentaires.
Recommandation 8. Que la pratique de consulter le personnel et de le faire participer à la planification et à l'exécution des programmes de recherche soit intensifiée et intégrée en profondeur à la culture de la haute direction de Forêts Canada.

Réponse. Forêts Canada accepte cette recommandation.

Mesure prise. Forêts Canada continue de promouvoir la participation du personnel à la planification et à l'exécution de la recherche. Dans une très large mesure, il en a toujours été ainsi au niveau des projets de recherche et des études, mais ce n'est que plus récemment qu'on a commencé à faire appel à cette pratique au niveau de l'identification stratégique des priorités. Les exemples les plus flagrants sont les nouvelles initiatives de recherche axée sur l'environnement prises en vertu du Plan vert. Forêts Canada continuera d'appuyer et de promouvoir cette tendance qui est très conforme à la philosophie de gestion du personnel du gouvernement fédéral qui est énoncée dans Fonction publique 2000. Simultanément, le Ministère est conscient qu'il lui faudra rester vigilant et gérer avec soin la participation des employés afin de s'assurer que soient en place des mécanismes leur permettant de contribuer de façon significative sans surcharger les scientifiques de responsabilités administratives.

Recommandation 9. Que Forêts Canada fasse participer activement ses scientifiques d'élite à l'élaboration de stratégies et de plans nationaux et à la création de réseaux de recherche.

Réponse. Forêts Canada accepte cette recommandation.

Mesure prise. Cette recommandation et les mesures prises à cet égard par Forêts Canada découlent directement de la recommandation 8 . La création de réseaux de recherche, la mise sur pied de partenariats et l'élaboration de plans stratégiques touchant des domaines particuliers de la recherche forestière retiennent de plus en plus l'attention de Forêts Canada, tout comme la recherche de mécanismes appropriés et économiques pour atteindre les objectifs de recherche. On fait énormément appel aux chercheurs émérites pour diriger ces initiatives, pratique qui se poursuivra. 